\title{
A Synergy between Push-Pull Electronic Effect and Twisted Conformation for High-Contrast Mechanochromic AIEgens
}

Xiaoxuan Wang, ${ }^{1,}$ Chunxuan Qi, ${ }^{1,}$ " Zhiyuan Fu, ${ }^{3}$ Haoke Zhang, ${ }^{2}$ Jianguo Wang, ${ }^{4}$ Hai-Tao Feng, $1,{ }^{*}$ Kai Wang, ${ }^{3}$ Bo Zou, ${ }^{3,}$ * Jacky W. Y. Lam ${ }^{2}$ and Ben Zhong Tang ${ }^{2, *}$

${ }^{1}$ AIE Research Center, Shaanxi Key Laboratory of Phytochemistry, College of Chemistry and Chemical Engineering, Baoji University of Arts and Sciences, Baoji 721013, China

${ }^{2}$ Department of Chemistry, Hong Kong Branch of Chinese National Engineering Research Center for Tissue Restoration \& Reconstruction, Institute for Advanced Study, Department of Chemical and Biomedical Engineering, Division of Life Science, State Key Laboratory of Molecular Neuroscience, The Hong Kong University of Science and Technology, Clear Water Bay, Hong Kong, China

${ }^{3}$ State Key Laboratory of Superhard Materials, Jilin University, Changchun 130012, P. R. China

${ }^{4}$ College of Chemistry and Chemical Engineering, Inner Mongolia Key Laboratory of Fine Organic Synthesis, Inner Mongolia University, Hohhot 010021, P. R. China.

Corresponding authors: Hai-Tao Feng (haitaofeng907@163.com), Bo Zou (zoubo@jlu.edu.cn), Ben Zhong Tang (tangbenz@ust.hk) 


\begin{abstract}
:
Mechanochromic (MC) luminogens in response to external stimulus have shown promising applications as pressure sensors and memory devices. While, research on their underlying mechanism is still in the initial stage. Here, three pyridinium-functionalized tetraphenylethylenes bearing $n$ pentyloxy group, hydrogen, nitro group, namely TPE-OP, TPE-H and TPE-NO, are designed to systematically investigate the influence of push-pull electronic effect and molecular conformation on MC luminescence. Upon anisotropic grinding and isotropic hydrostatic compression, TPE-OP with strong intramolecular charge transfer (ICT) affords the best MC behavior among them. Analysis of three polymorphs of TPE-H clearly indicates that planarization of molecular conformation is responsible for their bathochromic shift under mechanical stimuli. Theoretical calculations also verify that high twisting stress of AIEgens can be released under high pressure. This study presents a clear picture for $\mathrm{MC}$ behaviour and proposes an effective strategy to achieve high-contrast $\mathrm{MC}$ luminescence.
\end{abstract}




\section{Introduction}

Mechanochromic (MC) luminogens are pressure-responsive materials and show properly optical change in response to external stimuli, such as shearing, grinding, crushing, rubbing and pressing. ${ }^{1-4}$ MC materials, including organic compounds, ${ }^{5}$ polymers ${ }^{6}$ and metal complexes ${ }^{7}$, have attracted increasing interests over the past decades owing to their academic significance and promising applications as pressure sensors and memory devices. ${ }^{8-14}$ Pressure is a common external energy input and can induce MC luminescence through altering the chemical structure or solid-state morphology. ${ }^{15-18}$ Although the former mechanism is a universal method to achieve emission change in the solid state, scattered examples based on this strategy have been documented due to their irreversibility. ${ }^{19-23} \mathrm{Up}$ to now, most reported MC luminogens with red-shifted emission ${ }^{24-28}$ or blueshifted emission ${ }^{29,30}$ are attained by modulation of their morphologies, including transformation from crystal to crystal, ${ }^{31}$ crystal to amorphous state ${ }^{32,33}$ or stable to metastable phase in the liquid crystal, ${ }^{34}$ and conformation variation before and after exerting external stimuli. ${ }^{35,36}$ Although their mechanisms are somewhat clear case by case, they are not applicable in other systems. Thus, further deep understanding of the underlying mechanism and proposing an effective and general strategy for synthesizing MC luminogens will be of importance to guide molecular design and shed light on the relationship between molecular structure/packing and corresponding MC luminescence.

Generally, mechanical stimuli can be divided into anisotropic pressure (eg. grinding and rubbing) and isotropic compression (eg. hydrostatic pressure). ${ }^{37-42}$ In recent years, MC luminescence has been widely investigated by anisotropic pressure because of its simple operation. However, it comes across the bottleneck restriction in the development because it can only afford qualitative analysis of color change. To remove the constrain, big progress has been made by using isotropic compression through the diamond anvil cell (DAC) technique, which can realize in-situ monitoring the dynamic luminescence variation during the compression process. ${ }^{43,44}$ Unfortunately, due to high requirement for the instrument, only limited hydrostatic pressure-induced MC luminogens have been developed by Yamaguchi, ${ }^{45} \mathrm{He},{ }^{46} \mathrm{Zou},{ }^{47}$ and so on. These studies show that the hydrostatic pressure is a more useful and controllable tool to explore the structure-property relationship of MC materials than anisotropic pressure. Thus, much attention should be paid to this new technique.

Most MC luminogens prepared so far possess planar structure and show weak and low-contrast MC luminescence in the aggregated state due to aggregation-caused quenching (ACQ) effect, which dramatically impedes their real-world applications. Recently, a series of propeller-like molecules 
were found to display a phenomenon exact opposite of the ACQ effect. ${ }^{48-53}$ These molecules are non/weakly emissive in the molecularly dissolved state but fluoresce strongly in the condensed state. Such a photophysical phenomenon is termed as aggregation-induced emission (AIE). ${ }^{54-56}$ Accordingly, AIE luminogens (AIEgens) are decent alternatives to traditional dyes if they show MC behavior as well. The twisted conformations of AIEgens often afford a loose packing structure in the crystal state, which is beneficial for transformation between different phases through applying external stimuli. To date, some MC luminogens based on AIEgens have been reported by Chi, ${ }^{57}$ Tian, ${ }^{58} \mathrm{Li},{ }^{59}$ and so forth. While, for a better understanding of the molecular behavior under pressure, much more examples showing high contrast in emission switching and efficient solid-state luminescence need to be developed.

In this contribution, a group of pyridinium-functionalized tetraphenylethylene (TPE) luminogens with different substituents, namely TPE-OP, TPE-H and TPE-NO, were rationally designed and synthesized to explore the influence of molecular conformation and push-pull electronic effect on the MC luminescence in the crystalline state under mechanical stimuli. (Fig. 1). Through tuning the substituent from electron-donating $n$-pentyloxy group to electron-withdrawing nitro group, the resulting molecule exhibits quite different $\mathrm{MC}$ behaviours to its parent in response to anisotropic and isotropic pressure. Grinding of crystalline TPE-OP and TPE-H powders cause a remarkable luminescence red-shift. However, the electron-withdrawing character of the nitro group of TPE-NO weakens push-pull electronic effect to result in a minor emission red-shift in the presence of external force. These results indicate that AIEgens showing strong intramolecular charge transfer (ICT) characteristics are more susceptible to mechanical stimuli. Isotropic pressure was then applied to TPE-OP and TPE-H crystals to decipher the structure-property relationship. TPE-OP crystal shows a reversible emission switching between $562 \mathrm{~nm}$ and $675 \mathrm{~nm}$ with high contrast by repeated compression or decompression process. Meanwhile, single crystal of TPE-H exhibits an emission alteration from $479 \mathrm{~nm}$ to $529 \mathrm{~nm}$ under hydrostatic pressure. Upon relaxing the pressure, the emission cannot restore to the original state due to the hysteresis effect. By virtue of this effect, another two polymorphic crystals of TPE-H were cultured by using the compressed samples in the same tube with different standing time. These polymorphs emit much redder fluorescence than the pristine one due to their more planar molecular conformations, indicating that planarization of the twisted conformations of AIEgens is the main reason for their MC luminescence under mechanical stimuli. 


\section{Results}

Molecular engineering plays an important role in materials science and pharmaceutical study. The establishment of a large library of AIEgens will undoubtedly open a new venue to unravel the underlying mechanism of MC luminescence. Then, we adopt the following molecular design principles to achieve efficient MC AIEgens: 1) introduction of TPE can bestow the resulting molecules with AIE characteristics and loose molecular packing in the aggregated state by virtue of their twisted conformations. 2) It has been found that molecules with donor-acceptor (D-A) structure are easy to undergo conformation change under mechanical force. Thus, pyridinium was employed as a typical electron-withdrawing unit and coupled with electron-donating TPE to form a D-A structure with push-pull electronic effect. 3) Hexafluorophosphate anion can not only restrict the intramolecular motions but also inhibit $\pi-\pi$ interactions through anion $-\pi^{+}$interactions to further enhance solid-state emission efficiency. As shown in Fig. 1A, to strengthen the ICT transition, precursors 1A-1C were ionized by methyl iodide firstly. Then through anion exchange by hexafluorophosphate, three novel AIEgens (namely, TPE-OP, TPE-H and TPE-NO) with intrinsic positive charges were obtained. The two $n$-pentyloxy groups of TPE-OP further enhance the electrondonating power of TPE to afford strong ICT effect. On the other hand, TPE-NO bearing two nitro groups were designed as a control and TPE-H without any substituents was designed as an intermediate state. All the AIEgens were characterized satisfactorily by ${ }^{1} \mathrm{H},{ }^{13} \mathrm{C}$ and ${ }^{31} \mathrm{P}$ NMR and high-resolution mass spectrometry (Supplementary Fig. 19-S20). The detailed synthetic route and characterizations are presented in Scheme S1.

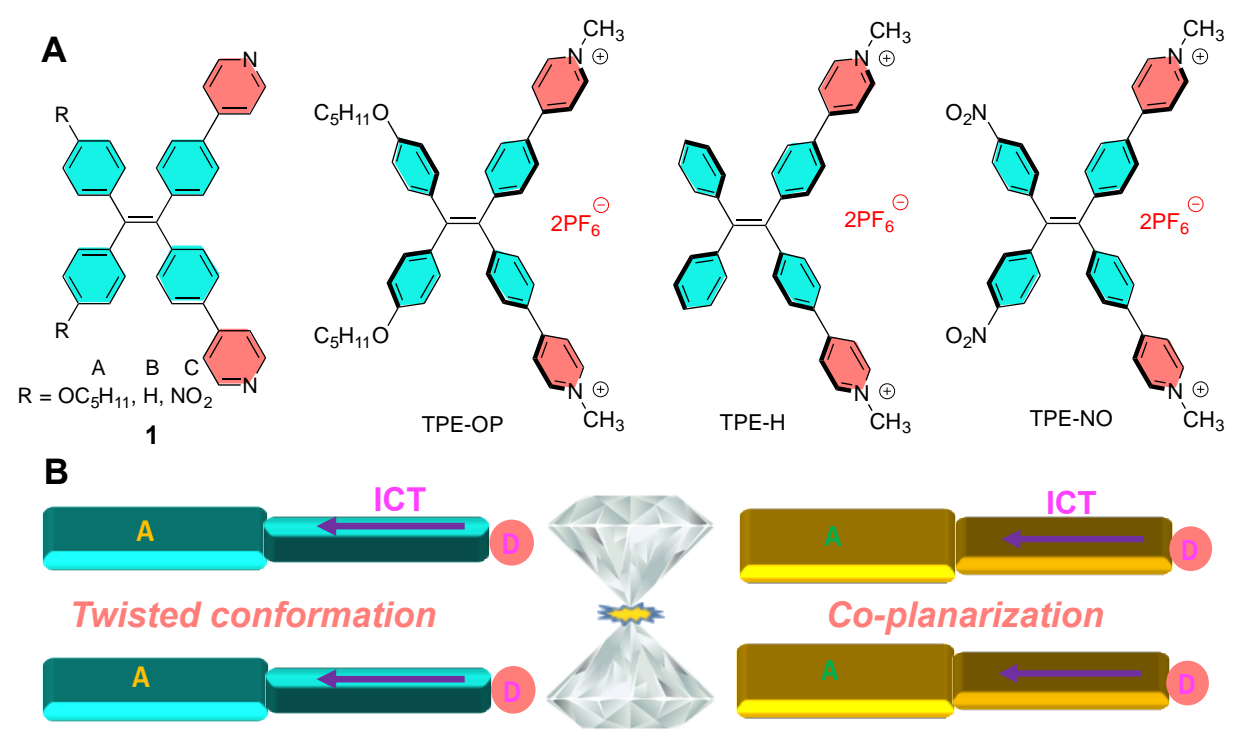

Fig. 1 Molecular structures and proposed mechanistic diagram. |(A) Molecular structures of precursor 1, TPEOP, TPE-H and TPE-NO. (B) Schematic illustration of work mechanism of MC behaviours. 
Photophysical Properties of TPE-OP, TPE-H and TPE-NO. The photoluminescence (PL) and UV-vis spectra of the molecules were measured in tetrahydrofuran (THF) solutions $\left(1.0 \times 10^{-5} \mathrm{M}\right)$ and aggregated state. TPE-OP showed a broad absorption band at $393 \mathrm{~nm}$ due to the ICT effect and a strong $\pi-\pi^{*}$ transition band at $285 \mathrm{~nm}$ in THF (Supplementary Fig. 1). Similar phenomena were also observed in TPE-H and TPE-NO with absorption bands at 355/283 nm and 340/280 nm, respectively. The absorption shift of TPE-OP > TPE-H > TPE-NO was attributed to the reinforced push-pull electronic effect. Upon photoexcitation, TPE-OP showed yellow emission in the aggregated state (Supplementary Fig. 2). The emission varied with the solvent polarity owing to the ICT effect. Their AIE characteristics were confirmed in THF/hexane mixtures. Upon addition of poor solvent hexane to THF, weak fluorescence of TPE-OP was observed with a low QY of $1.2 \%$ at the hexane fraction less than $60 \%$ (Supplementary Fig. 3). At $80 \%$ hexane fraction, the PL intensity was drastically boosted. By further enhancing the fraction to $95 \%$, slightly decrease in PL intensity occurred due to the generation of amorphous aggregates. The solid-state QY of TPE-OP measured by using an integrating sphere was $86.2 \%$, being 72 -fold higher than that in solution. Clearly, TPEOP is AIE-active. TPE-H was also AIE-active and its QY enhanced from $0.9 \%$ in solution to $31.2 \%$ in solid (Supplementary Fig. 4). The AIE property of TPE-NO was similar to TPE-OP, it was weakly emissive in THF $\left(\Phi_{\text {soln }}=0.8 \%\right)$, but fluoresced strongly in $70 \% \mathrm{THF} /$ hexane fraction. The PL intensity, however, decreased slightly at higher hexane fraction (Supplementary Fig. 5). The QY of TPE-NO in the solid state was calculated to be $12.8 \%$.

MC Behaviours upon Anisotropic Grinding. To evaluate the MC behaviours of the AIEgens, their solid-state emission was explored in the presence and absence of anisotropic grinding. Our preliminary research indicates that precursor $\mathbf{1 A}$ is an force-responsive luminogen. ${ }^{37}$ Upon ionization, as shown in Fig. 2 and Supplementary Fig. 6, the pristine powders of TPE-OP and TPE-H emitted bright yellow and greenish yellow fluorescence at $563 \mathrm{~nm}$ and $516 \mathrm{~nm}$, respectively $(\mathrm{QY}(\Phi)=86.2 \%$, lifetime $(\tau)=4.07 \mathrm{~ns}$ for TPE-OP; $\Phi=31.2 \%, \tau=3.08 \mathrm{~ns}$ for TPE-H). Upon grinding with a pestle, TPE-OP emitted red PL with a $60 \mathrm{~nm}$ red-shift in the emission maximum. The fluorescence QY of TPE-OP became lower (56.4\%) but the lifetime was enhanced to 5.56 ns (Supplementary Fig. 7). For TPE-H, its PL was red-shifted to $554 \mathrm{~nm}$ to display a wavelength change of $38 \mathrm{~nm}$. The QY and lifetime of TPE-H presented similar trend as those of TPE-OP $(\Phi=22.1 \%, \tau=5.40 \mathrm{~ns})$ (Supplementary Fig. 8), suggesting that anisotropic grinding drastically affects its emission behaviour. To further understand the photophysical properties of TPE-OP and TPE-H, their fluorescence rate 
constants were calculated and summarized in Table 1 . The radiative decay constants $\left(k_{\mathrm{r}}\right)$ of TPE-OP and TPE-H from $\mathrm{S}_{1}$ to $\mathrm{S}_{0}$ decreased from $2.11 \times 10^{8}$ and $1.01 \times 10^{8} \mathrm{~s}^{-1}$ in pristine powders to $1.01 \times$ $10^{8}$ and $0.41 \times 10^{8} \mathrm{~s}^{-1}$ after grinding, respectively. The non-radiative rate constants $\left(k_{\mathrm{nr}}\right)$, however, only enhanced in a small extent, which implied that the emission efficiency became weaker in the presence of anisotropic force. Then, UV-vis measurements were carried out to explore their groundstate change. As shown in Fig. 2B and Supplementary Fig. 6, TPE-OP and TPE-H showed obvious red-shifted absorption upon grinding, which was consistent with the distinct PL spectrum shift. On the other hand, the emission of the ground powders could recover to their original state by solvent fuming, indicating the reversibility of the MC luminescence process. Powder X-ray diffraction (PXRD) was then implemented to understand the relationship of PL variation and molecular packing. The PXRD diffractograms of pristine powder of TPE-OP and TPE-H exhibited many sharp and strong scattering peaks, suggestive of their good crystallinity with ordered molecular packing (Fig. 2D and Supplementary Fig. 9A). However, the ground powder showed only broad and weak peaks, implying the occurrence of phase transition from crystalline to amorphous state upon grinding.
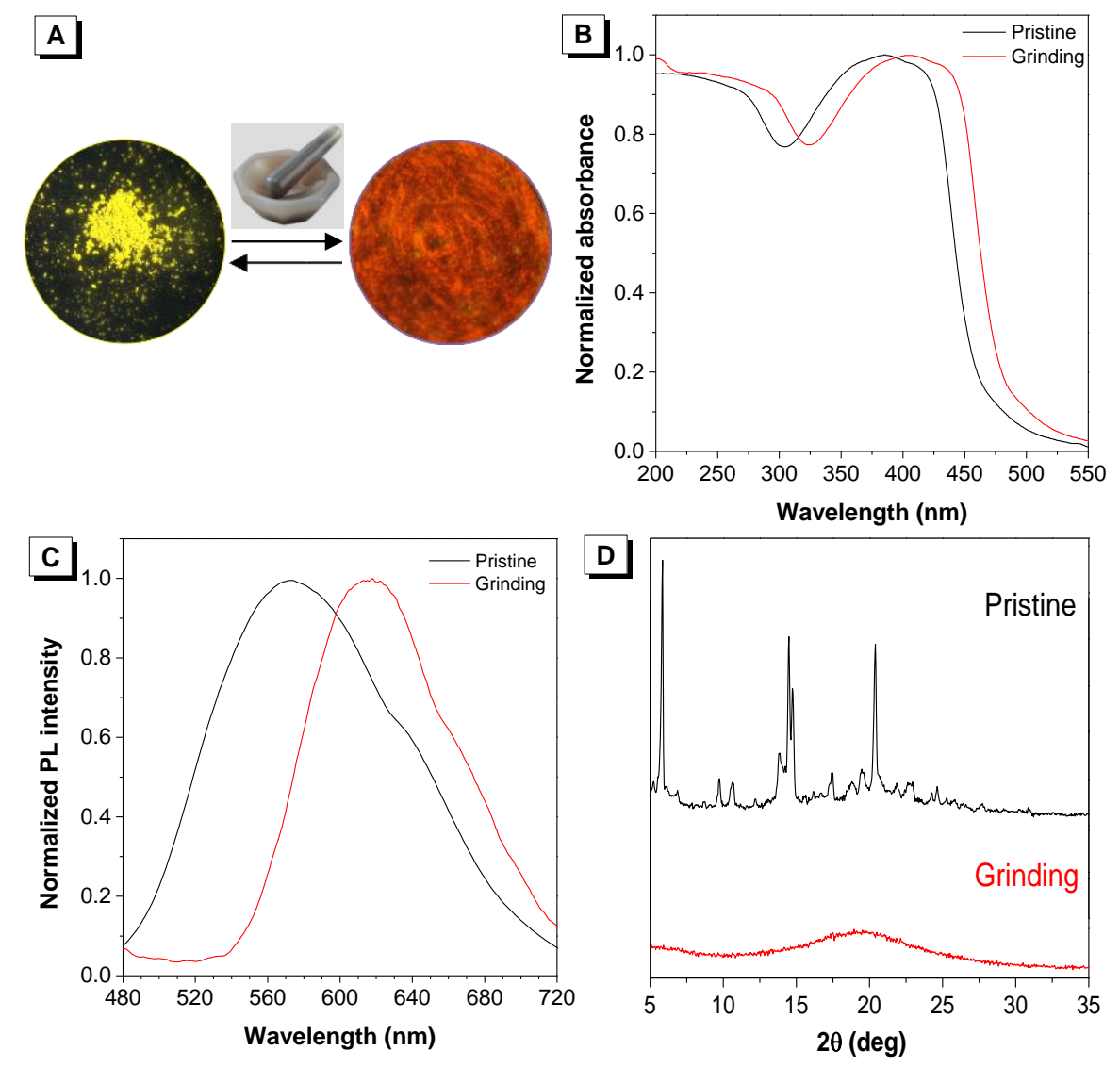

Fig. 2 The luminescent images, PL spectra and PXRD patterns. | (A) Photos of reversible mechanochromic luminescence of TPE-OP taken under $365 \mathrm{~nm}$ UV irradiation. (B) UV-vis and (C) PL spectra of TPE-OP before and after grinding. Exitation wavelength: $380 \mathrm{~nm}$. (D) PXRD patterns of TPE-OP before and after grinding. 
Table 1. Photophysical Properties of TPE-OP, TPE-H and TPE-NO

\begin{tabular}{|c|c|c|c|c|c|c|c|c|c|c|c|}
\hline & \multirow{2}{*}{$\frac{\lambda_{\mathrm{abs}}(\mathrm{nm})}{\text { solution }}$} & \multirow{2}{*}{$\frac{\lambda_{\mathrm{em}}(\mathrm{nm})}{\text { pristine }}$} & \multicolumn{3}{|c|}{$\Phi(\%)^{a}$} & \multicolumn{2}{|c|}{$\tau(\mathbf{n s})^{\mathbf{b}}$} & \multicolumn{2}{|c|}{$k_{\mathrm{r}}\left(\times 10^{8} \mathrm{~s}^{-1}\right)^{\mathrm{c}}$} & \multicolumn{2}{|c|}{$k_{\mathrm{nr}}\left(\times 10^{8} \mathrm{~s}^{-1}\right)^{\mathrm{d}}$} \\
\hline & & & solution & pristine & ground & pristine & ground & pristine & ground & pristine & ground \\
\hline TPE-OP & 393 & 563 & 1.2 & 86.2 & 56.4 & 4.07 & 5.56 & 2.11 & 1.01 & 0.34 & 0.78 \\
\hline TPE-H & 355 & 516 & 0.9 & 31.2 & 22.1 & 3.08 & 5.40 & 1.01 & 0.41 & 1.23 & 1.44 \\
\hline TPE-NO & 340 & 497 & 0.8 & 12.8 & 10.9 & 3.17 & 3.40 & 0.40 & 0.26 & 2.75 & 2.68 \\
\hline
\end{tabular}

In contrast, TPE-NO with a A-A structure was not sensitive to anisotropic grinding due to the intrinsic electron-withdrawing feature of nitro group. The pristine powder of TPE-NO emitted a green colour at $497 \mathrm{~nm}$ with a fluorescence QY of $12.8 \%$ and lifetime of $3.17 \mathrm{~ns}$ (Supplementary Fig. 10). Upon grinding, the PL spectrum was shifted by $19 \mathrm{~nm}$ to $516 \mathrm{~nm}$, whose wavelength change was much smaller than TPE-OP and TPE-H. The fluorescence QY and lifetime also exhibited a minor variation after grinding $(\Phi=10.9 \%, \tau=3.40 \mathrm{~ns})$ (Supplementary Fig. 11). The $k_{\mathrm{r}}$ of TPE-NO decreases from $0.40 \times 10^{8}$ to $0.26 \times 10^{8} \mathrm{~s}^{-1}$ and the $k_{\mathrm{nr}}$ also exhibits a negligible change. The PXRD patterns verified that amorphous aggregates were generated by grinding as well (Supplementary Fig. 9B). The above results clearly demonstrated that the MC behaviors of AIEgens were largely influenced by the push-pull electronic effect and a D-A structure is favourable to show an enhanced MC performance.

MC Behaviours upon Isotropic Pressure. On the other hand, hydrostatic pressure as a common isotropic compression is often applied on single crystals to in-situ monitor the MC process. The pressure-dependent MC experiments were investigated by a DAC technique at room temperature. The single crystal of TPE-OP was yellow in colour with good optical transparency under day light and ambient pressure (Fig. 3A). However, its colour turned to orange and then deep red with increasing the surrounding pressure. As discussed above, TPE-OP emitted intense yellow fluorescence at $562 \mathrm{~nm}$ at normal atmosphere pressure. When the pressure was raised to $0.53 \mathrm{GPa}$, its PL spectrum was red-shifted to $585 \mathrm{~nm}$ accompanied with a decreased intensity (Fig. 3B). Further raising pressure to $4.06 \mathrm{GPa}$, red emission at $622 \mathrm{~nm}$ was attained, which was similar to that of the ground crystals (Supplementary Fig. 12). When the pressure was further increased to $10.04 \mathrm{GPa}$, the emission band gradually shifted to $675 \mathrm{~nm}$ and its intensity continuously decreased. This indicated that hydrostatic pressure was more powerful to generate intense MC luminescence. Generally, pressure-responsive luminogens afford a planar conformation at high pressure to enhance the 
oscillator strength to cause PL attenuation. ${ }^{34}$ On the other hand, the large wavelength change of 113 $\mathrm{nm}$ indicated high-contrast emission was achieved by hydrostatic pressure. In the following decompression process, the emission wavelength could be restored to the original state, but the emission intensity suffered a certain decline (Fig. 3C). The high reversibility of the MC behaviors implied that the molecular phase and packing mode underwent no remarkable change during compression. Thus, it was assumed that the MC luminescence of TPE-OP under hydrostatic pressure was ascribed to the planarization of molecular conformation. As a structural evidence, dynamic Raman spectroscopy was performed on TPE-OP crystal (Fig. 3D). Some stretching and bending vibrations of TPE-OP disappeared at pressure above 4.97 GPa and obvious blue-shift was observed in the range of $900-1120 \mathrm{~cm}^{-1}$ from the amplified Raman spectra (Supplementary Fig. 13). These results indicated that hydrostatic pressure-induced conformational planarization was attributed to the MC luminescence of propeller-like AIEgens.
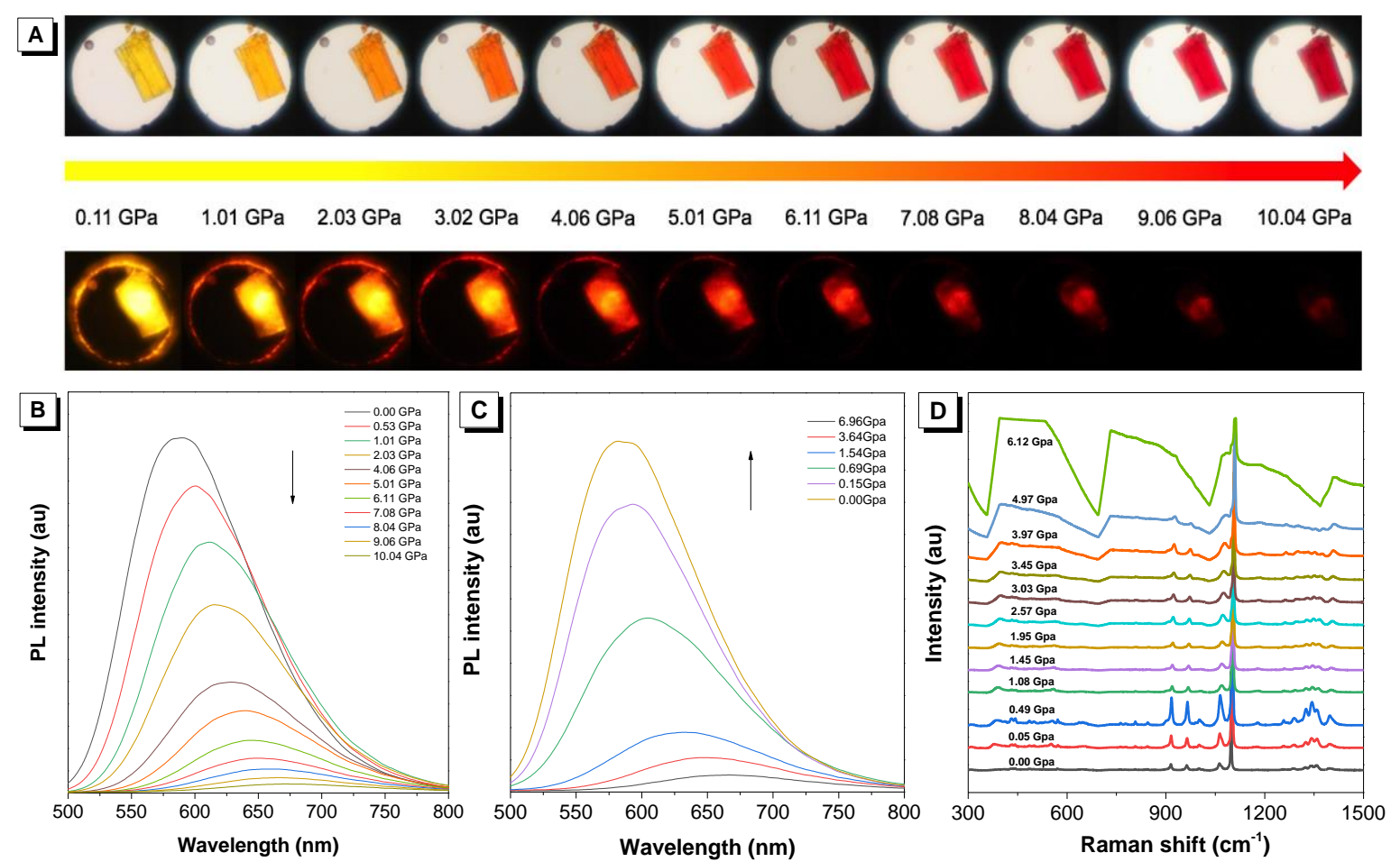

Fig. 3 PL properties of TPE-OP under hydrostatic pressure. | (A) In-situ images of TPE-OP crystal taken under daylight and UV light. In-situ PL spectra of TPE-OP crystal measured at (B) increasing pressure and (C) decreasing pressure. (D) In-situ Raman spectra taken at increasing pressure.

TPE-H is colorless with excellent transparency at ambient pressure (Fig. 4A). Upon application of hydrostatic pressure $(10.00 \mathrm{GPa})$ to its single crystal, the color turned to brown. The pristine crystal of TPE-H showed a bright sky-blue emission at $479 \mathrm{~nm}$ under UV irradiation. At high pressure, a 
notable colour change from blue to green and then to greenish yellow was observed, suggesting that the molecular planarity was gradually improved (Fig. 4C). By further raising the pressure to 10.00 $\mathrm{GPa}$, the fluorescence intensity decreased dramatically and a broad band centered at $529 \mathrm{~nm}$, implying that the $k_{\mathrm{r}}$ value reduced remarkably during compression. To confirm the molecular conformation alteration, in situ UV-vis measurement was performed at different pressures. As shown in Fig. 4B, the UV-vis spectrum of TPE-H showed minor change at pressure less than $6.03 \mathrm{GPa}$. However, continuously increasing the pressure to $10.00 \mathrm{GPa}$, the absorption band red-shifted to the long visible wavelength, which was in agreement with the noticeable red-shift of the PL spectrum. When the pressure was released to $0 \mathrm{GPa}$, the emission could only restore to $491 \mathrm{~nm}$ even the sample was stood for several days (Fig. 4D). Such a emission wavelength was also attained when the sample was compressed at $1.98 \mathrm{GPa}$. Such spectral residue may be ascribed to the hysteresis effect that the phenyl rings of TPE-H suffer from difficulty in recovering to their original position owing to the possible structural deformation at high pressure. The Raman spectrum of TPE-H blue-shifted with increasing pressure, demonstrating the occurrence of structural variation in the crystal (Supplementary Fig. 14).
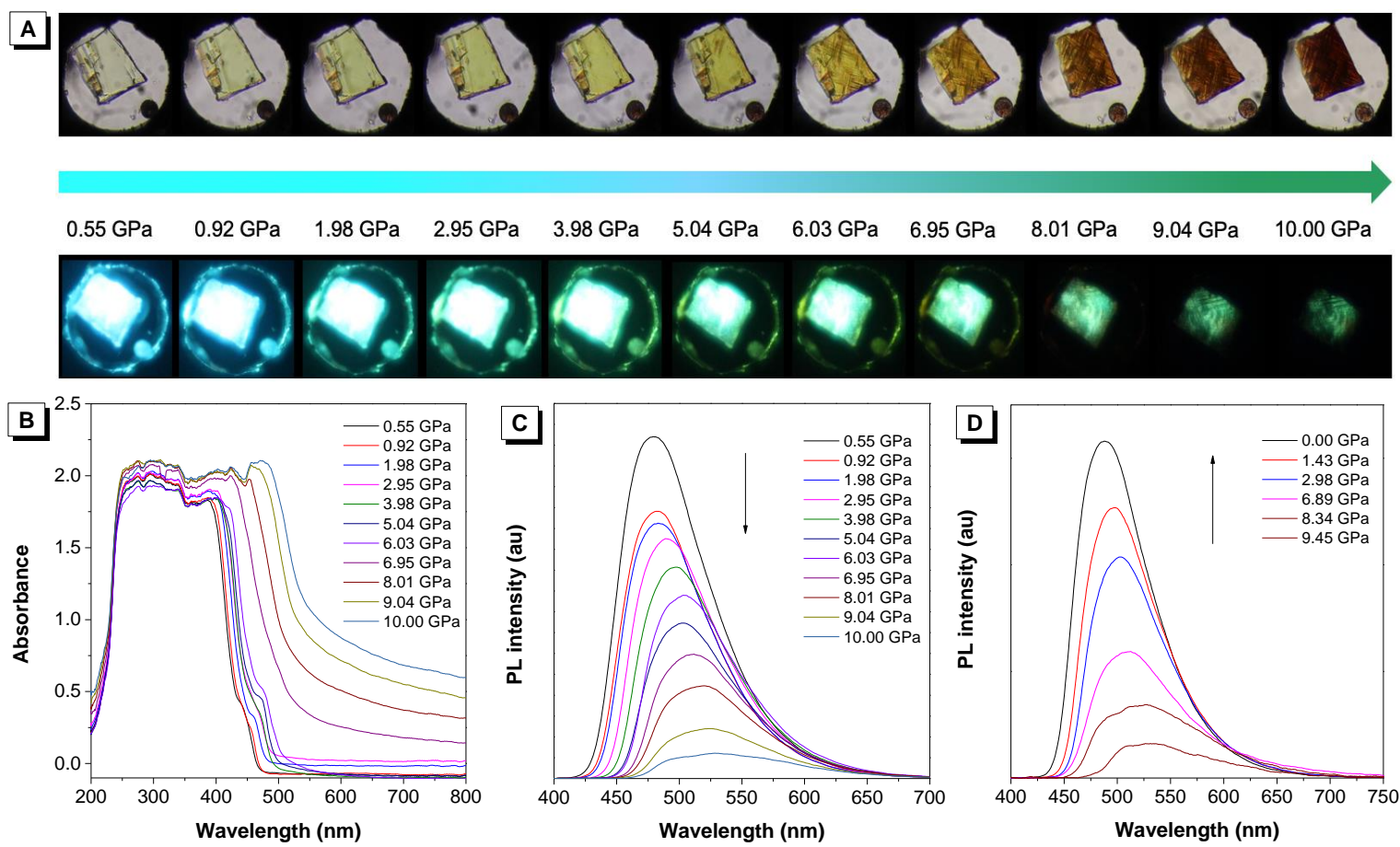

Fig. 4 PL properties of TPE-H under hydrostatic pressure. | (A) In-situ images of TPE-H crystal taken under daylight and UV light. In-situ (B) UV-Vis and (C) PL spectra of TPE-H crystal measured during the compression process. (D) In-situ PL spectra of TPE-H crystal during releasing pressure. 
Analysis of Single Crystals. By virtue of this hysteresis effect, an attempt was made to obtain single crystals with planarized conformation by using compressed samples. Fortunately, another two polymorphic crystals of TPE-H suitable for X-ray structural analysis were cultured by slow evaporation of a THF solution of its compressed samples in the same tube with different standing time. Upon evaporation of the solution after two days, a blocky crystal of TPE-H (namely TPE-H-K) displaying greenish-yellow emission was formed. When elongating the standing time to 14 days, another rod-like crystal emitting green light was achieved (Fig. 5A). PL measurement showed that pristine crystal of TPE-H, TPE-H-K, TPE-H-T and crystalline powder of TPE-H exhibited emission bands at $479 \mathrm{~nm}, 506 \mathrm{~nm}, 496 \mathrm{~nm}$ and $516 \mathrm{~nm}$, respectively (Fig. 5B).
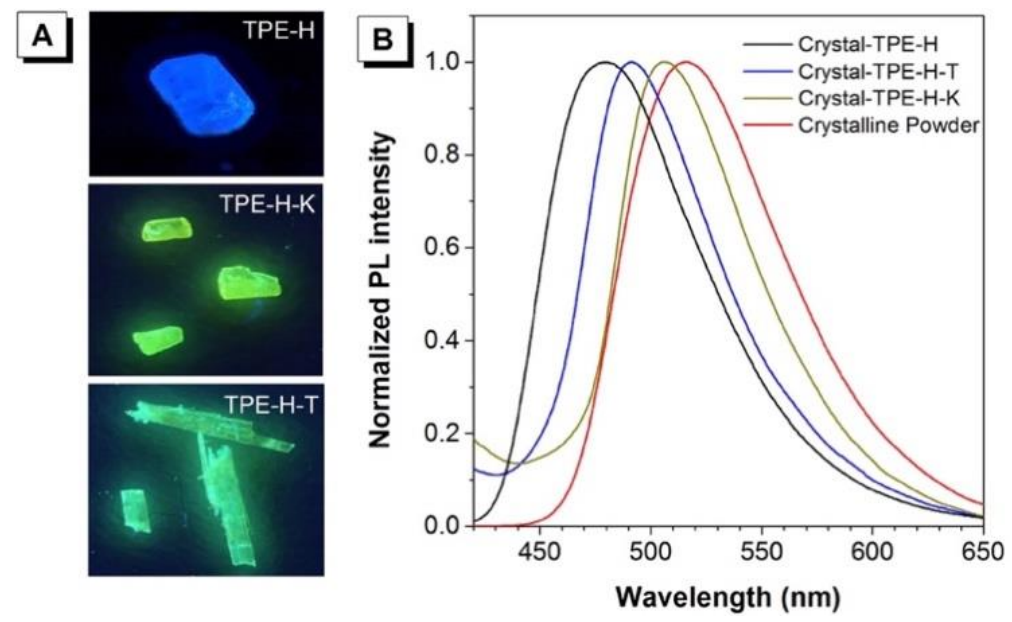

Fig. 5 PL properties of polymorphs of TPE-H. | (A) Fluorescent photos of crystal of TPE-H, TPE-H-K and TPEH-T taken under UV irradiation. (B) PL spectra of TPE-H in different crystalline morphologies.

\section{Discussion}

Such intriguing phenomenon motivate us to investigate the relationship between the MC luminescence and the molecular structure. First, the precursors (1A, 1B and 1C) were took as examples to cognize MC luminescence under anisotropic grinding. Upon grinding of $\mathbf{1 A}, \mathbf{1 B}$ and $\mathbf{1 C}$, they all showed obvious colour change from blue to greenish-yellow. Analysis by crystal structures showed that all the molecules adopt a twisted conformation with high twisting stress in crystals due to the strong steric hindrance of neighbouring phenyl rings. The torsion angles between phenyl rings and double bond of $\mathbf{1 A}$ were in the range of $39.75^{\circ}$ to $53.49^{\circ}$. Such large distortion impeded a compact $\pi-\pi$ stacking between adjacent molecules (Supplementary Fig. 15). Moreover, the long $n$-pentyloxy groups of $\mathbf{1 A}$ also served as blocks to afford a loose molecular packing. Besides, some short contacts, 
such as $\mathrm{C}-\mathrm{H} \cdots \mathrm{N}\left(d_{\mathrm{CH} \cdots \mathrm{N}}=2.683 \AA\right), \pi-\pi\left(d_{\pi \cdots \pi}=3.353 \AA\right)$ interactions, were observed in neighbouring molecules to solidify the molecular conformation. Precursor $\mathbf{1 B}$ also showed a twisted conformation with torsion angles lying between $39.18^{\circ}$ and $52.14^{\circ}$, and adopted a face-to-face stacking by the aid of numerous non-covalent interactions including $\mathrm{C}-\mathrm{H} \cdots \mathrm{N}\left(d_{\mathrm{CH} \cdots \mathrm{N}}=2.350 \AA\right), \mathrm{C}$ $\mathrm{H} \cdots \pi\left(d_{\mathrm{CH} \cdots \mathrm{N}}=2.822,2.825 \AA\right), \mathrm{C}-\mathrm{H} \cdots \mathrm{C}\left(d_{\mathrm{CH} \cdots \mathrm{N}}=2.862 \AA\right)$ and $\pi-\pi\left(d_{\pi \cdots \pi}=3.369 \AA\right)$ (Supplementary Fig. 16). The torsion angles of 1C fell in the scope of $37.34^{\circ}$ and $52.25^{\circ}$ and the neighboring molecules were contacted by $\mathrm{C}-\mathrm{H} \cdots \mathrm{N}\left(d_{\mathrm{CH} \cdots \mathrm{N}}=2.712 \AA\right), \mathrm{C}-\mathrm{H} \cdots \mathrm{O}\left(d_{\mathrm{CH} \cdots \mathrm{N}}=2.862 \AA\right)$ and $\pi-\pi\left(d_{\pi \cdots \pi}=3.359 \AA\right)$ interactions (Supplementary Fig. 17). The results gave a clear picture for AIE behaviour that when these luminogens were in the molecularly dissolved state, their excited-state energy were consumed non-radioactively through rotation or vibration to render them non-emissive characters. However, in the crystalline state, the multiple non-covalent interactions could efficiently inhibit intramolecular motions and rigidified further the molecular conformation. These decreased the energy loss by non-radiative pathway enabled the molecules to show intense emission. Upon amphorization by anisotropic grinding, the high twisting stress and weak interactions existing in crystals were destructed to generate a relatively planarized conformation, accompanied by a decreased QY and a redder emission.

Three polymorphs (TPE-H, TPE-H-K and TPE-H-T) were then utilized to systematically analyse the structure-property relationship under hydrostatic pressure. TPE-H and TPE-H-T crystals belonged to the monoclinic system, while, TPE-H-K crystallized in an orthorhombic manner. In the crystal state, TPE-H showed the most twisted molecular conformation as compared to TPE-H-K and TPE-H-T. As shown in Fig. 6, the torsion angles of TPE-H are $43.37^{\circ}\left(\theta_{1}\right), 58.02^{\circ}\left(\theta_{2}\right), 49.46^{\circ}\left(\theta_{3}\right)$, $48.66^{\circ}\left(\theta_{4}\right), 34.03^{\circ}\left(\theta_{5}\right)$ and $31.10^{\circ}\left(\theta_{6}\right)$, respectively. In contrast, TPE-H-K cultured from the compressed samples for two days exhibited a more effective conjugation with notably reduced torsion angles of $43.57^{\circ}\left(\theta_{1}\right), 47.98^{\circ}\left(\theta_{2}\right), 47.98^{\circ}\left(\theta_{3}\right), 43.57^{\circ}\left(\theta_{4}\right), 29.70^{\circ}\left(\theta_{5}\right)$ and $29.70^{\circ}\left(\theta_{6}\right)$. Such a planarized molecular conformation was attributed to the hysteresis effect at high hydrostatic pressure and was also responsible for its greenish-yellow emission at $506 \mathrm{~nm}$. As extended the standing time, the asprepared TPE-H-T crystals were apt to recover to its original state and presented a more twisted conformation than TPE-H-K. The corresponding torsion angles increased to $46.02^{\circ}\left(\theta_{1}\right), 54.14^{\circ}\left(\theta_{2}\right)$, $54.14^{\circ}\left(\theta_{3}\right), 46.02^{\circ}\left(\theta_{4}\right), 32.91^{\circ}\left(\theta_{5}\right)$ and $32.91^{\circ}\left(\theta_{6}\right)$, respectively. Consequently, the reduction of molecular conjugation lead to green fluorescence at $496 \mathrm{~nm}$. Moreover, the two hexafluorophosphate anions were in close contact with the pyridinium core with distances of 3.18 and $3.20 \AA$ in TPE-H. 
This clearly demonstrated the existence of anion $-\pi^{+}$interactions, which can restrict the intramolecular motions and inhibit $\pi-\pi$ interactions between neighbouring molecules. However, the distances raised to $3.61 \AA$ in TPE-H-K, implying that the interactions became weakened. While, the distances shortened to $3.38 \AA$ in TPE-H-T. Due to the twisted conformation and large steric hindrance of hexafluorophosphate, the coplanar $\pi-\pi$ stacking was impossible to be formed in these AIEgens. Crystals of TPE-H and TPE-H-T exhibited a head-to-tail molecular packing mode with strong short contacts of $\mathrm{C}-\mathrm{H} \cdots \pi, \mathrm{F}-\pi$ and $\mathrm{C}-\mathrm{H} \cdots \mathrm{F}$. In contrast, the TPE-H-K crystal adopted a head-to-head packing assisted by only relatively loose $\mathrm{F}-\pi$ and $\mathrm{C}-\mathrm{H} \cdots \mathrm{F}$ interactions. These solid evidences clearly demonstrated that planarization of the twisted molecular conformation under hydrostatic pressure caused the red-shifted emission and sufficient intermolecular interactions can rigidify the molecular conformation to give rise to a high emission efficiency in the crystalline state.
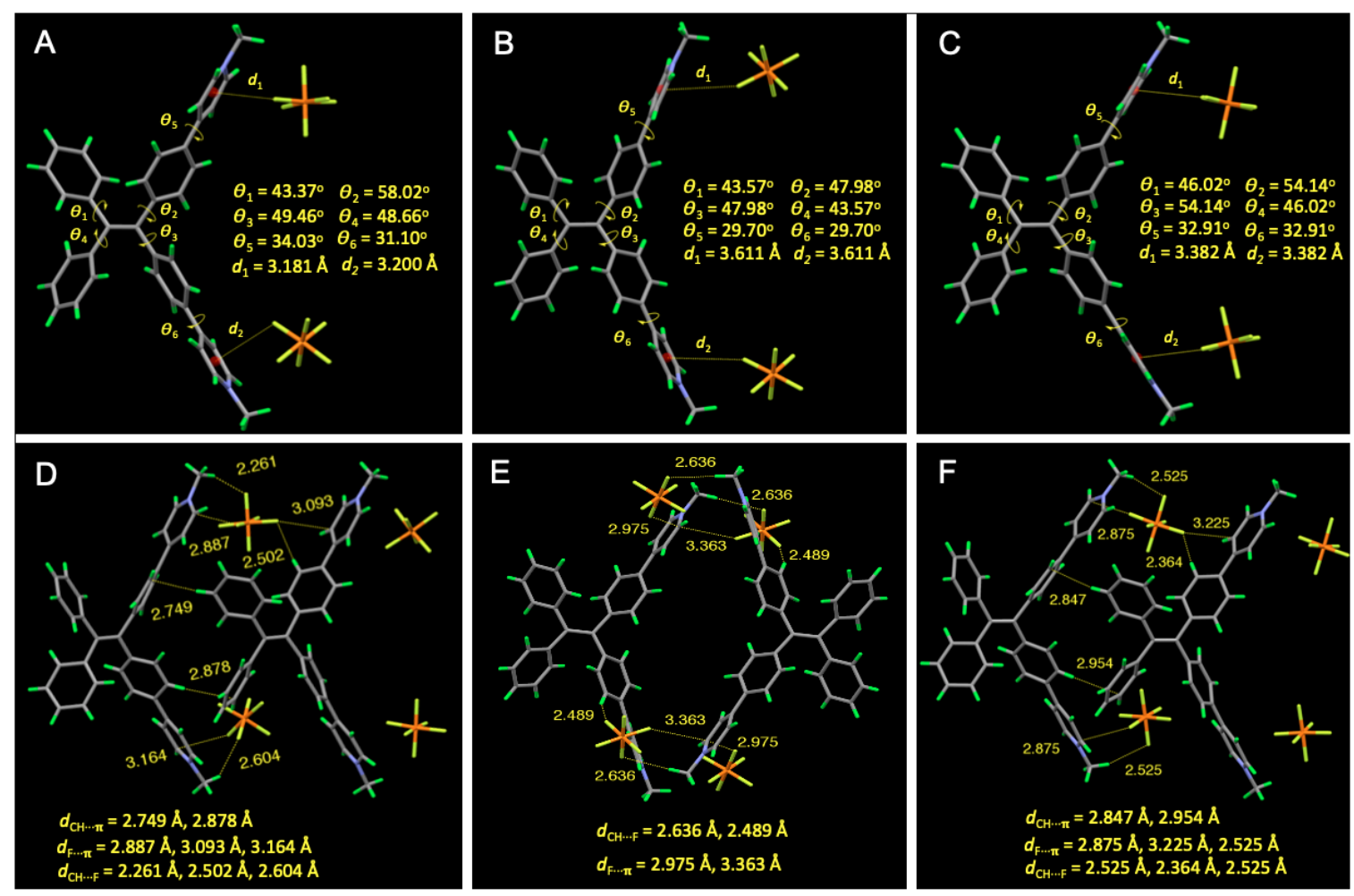

Fig. 6 Crystal structures of polymorphs. | The molecular geometry and intermolecular interactions of (A and D) TPE-H, (B and E) TPE-H-K and (C and F) TPE-H-T in crystals. Green: Hydrogen, Grey: Carbon, Yellow: Fluorine, Orange: Phosphorus and Purple: Nitrogen.

To further understand the MC behavior, time-dependent density functional theory (TD-DFT) simulations were carried out based on the single crystals at the B3LYP/6-311G** level. As depicted in Supplementary Fig. 18, the vertical excitation energies were calculated first under different torsion angles. In each step, only the chosen torsion angle was immobilized and all other atoms were fully 
relaxed to exert the geometric optimization. As an increment of torsion angle of C1-C2-C3-C4 in TPE-OP from 0 to $60^{\circ}$, the excitation energy decreased from $0.32 \mathrm{eV}$ to the minimum at $25^{\circ}$, but it gradually raised up to $0.19 \mathrm{eV}$ at $60^{\circ}$. When the torsion angle of C3-C4-C5-C6 was flipped, its excitation energy increased initially from 0 to $50^{\circ}$, but showed a small decease at $60^{\circ}$. However, the energy change $(0.055 \mathrm{eV})$ was quite small as compared to the value of $\mathrm{C} 1-\mathrm{C} 2-\mathrm{C} 3-\mathrm{C} 4$. This suggested that the rotation of $n$-pentyloxy substituted phenyl rings played a minor role in tuning the excitation energy. Through analysis of the excitation energy of TPE-H under different torsion angles, both kinds of rotations (C1-C2-C3-C4 and C3-C4-C5-C6) were found to be important to achieve a low excitation energy. Taking these factors into consideration, it evidently indicated that the relatively small torsion angles between phenyl rings and double bond were beneficial to give a low vertical excitation energy and enhance co-planarity of the whole molecule to lead to red-shifted emission. Fig. 7 depicts the frontier orbitals of the polymorphs of TPE-H. The highest occupied molecular orbital (HOMO) of all polymorphic crystals was mainly resided on the phenyl rings and double bond. The lowest unoccupied molecular orbital (LUMO), on the other hand, was primarily localized on the pyridinium groups due to their electron-withdrawing nature. Upon excitation, electrons were transferred from the phenyl rings of TPE to the pyridinium groups. Thus, an effective CT transition occurred by virtue of the spatial separation of HOMO and LUMO. Due to different molecular conformations, the calculated energy gap were in the order of TPE-H $(2.339 \mathrm{eV})>$ TPE-H-T $(2.290 \mathrm{eV})>$ TPE-H-K $(2.265 \mathrm{eV})$, which was consistent with their PL spectra. Moreover, the vertical excitation energy of $\mathrm{S}_{1}$ also showed an order similar to that of energy gap, further verifying the above-mentioned results.

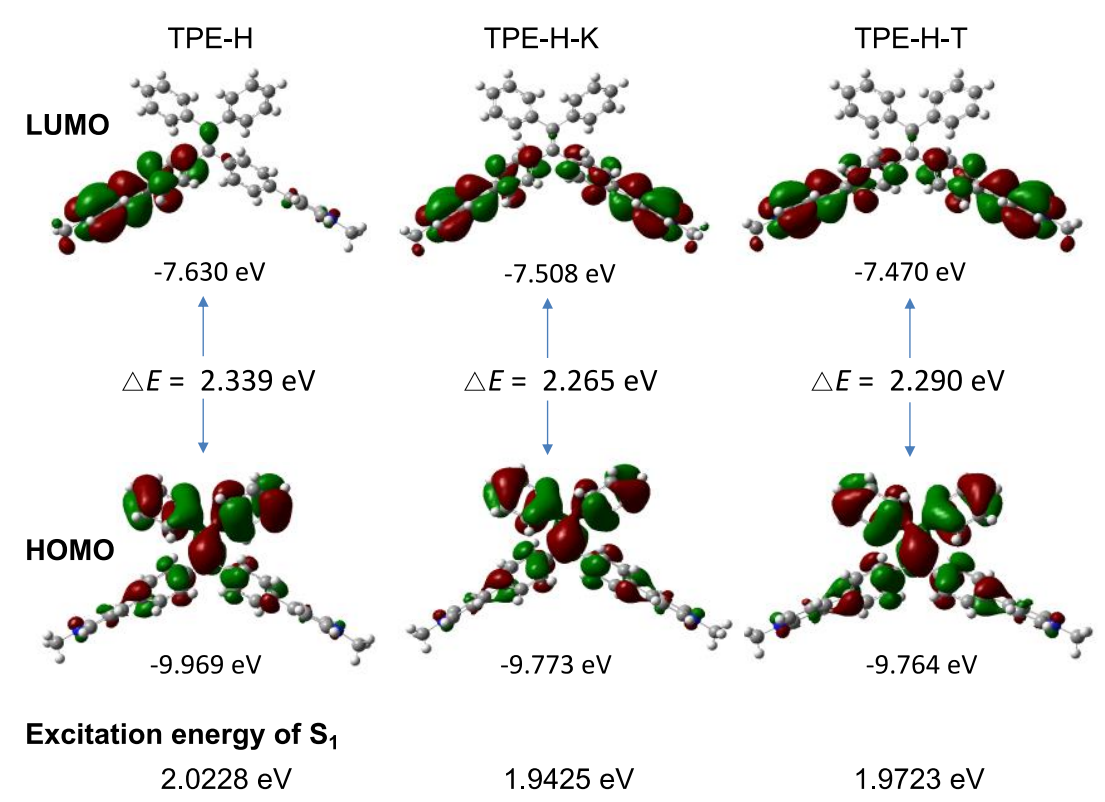

Fig. 7 Calculated energy diagram. | Molecular orbitals and vertical excitation energies of polymorphs of TPE-H. 
In this work, three pyridinium-functionalized TPE derivatives with different substituents were designed and synthesized. Through anion exchange, two hexafluorophosphate anions were introduced to construct anion $-\pi^{+}$interactions in these AIEgens to restrict the intramolecular motions and inhibit compact $\pi-\pi$ interactions between neighbouring molecules. Upon anisotropic grinding, TPE-OP with effective ICT transition showed the better MC characteristics than TPE-H and TPENO. Such emission alteration was reversible aided by solvent fuming. When isotropic hydrostatic pressure was applied on the crystals of TPE-OP and TPE-H, high-contrast MC emission was observed. In the following decompression process, the emission of TPE-H cannot recover to its original state due to the hysteresis effect. Taking advantage of this effect, another two polymorphs of TPE-H were obtained by using the compressed samples with different standing time. Careful investigation of their different emission properties found that the redder fluorescence of TPE-H-K was attributed to its much more planar molecular conformation than TPE-H and TPE-H-T. Similar results were also obtained from theoretical calculation. In a word, the ICT-type AIEgens with multiple intermolecular interactions and loose molecular packing mode are ideal candidates for high-contrast MC luminogens and planarization of molecular conformation is responsible for their emission variation under external stimuli.

\section{Data availability}

The data that support the findings of this study are available in Supplemenary Information and from the corresponding author on reasonable request.

\section{References}

1. Chi, Z. et al. Recent advances in organic mechanofluorochromic materials. Chem. Soc. Rev. 41, 3878-3896 (2012).

2. Ma; Z.; Wang, Z.; Teng M.; Xu, Z.; Jia, X. Mechanically Induced Multicolor Change of Luminescent Materials. ChemPhysChem 16, 1811-1828 (2015).

3. Xu, J.; Chi, Z.; Mechanochromic Fluorescent Materials: Phenomena, Materials and Applications. Royal Society of Chemistry (2014).

4. Dong, Y. Q.; Lam, J. W. Y.; Tang, B. Z. Mechanochromic Luminescence of Aggregation-Induced Emission Luminogens. J. Phys. Chem. Lett. 6, 3429-3436 (2015).

5. Wang, C.; Li, Z. Molecular conformation and packing: their critical roles in the emission performance of mechanochromic fluorescence materials. Mater. Chem. Front. 1, 2174-2194 (2017).

6. Ciardelli, F.; Ruggeri, G.; Pucci, A. Dye-containing polymers: methods for preparation of mechanochromic materials. Chem. Soc. Rev. 42, 857-870 (2013).

7. Zhang, X.; Chi, Z.; Zhang, Y.; Liu, S.; Xu, J. Recent advances in mechanochromic luminescent metal 
complexes. J. Mater. Chem. C 1, 3376-3390 (2013).

8. Mutai, T.; Satou, H.; Araki, K. Reproducible On-Off Switching of Solid-State Luminescence by Controlling Molecular Packing Through Heat-Mode Interconversion. Nat. Mater. 4, 685-687 (2005).

9. Sagara, Y.; Kato, T. Mechanically induced luminescence changes in molecular assemblies. Nat. Chem. 1, 605-610 (2009).

10. Gong, Y.-B. et al. The Influence of Molecular Packing on the Emissive Behavior of Pyrene Derivatives: Mechanoluminescence and Mechanochromism. Adv. Opt. Mater. 6, 1800198 (2018).

11. Sun, H. et al. Smart Responsive Phosphorescent Materials for Data Recording and Security Protection. Nat. Commun. 5, 3601 (2014).

12. Lavrenova, A. et al. Mechano- and Thermoresponsive Photoluminescent Supramolecular Polymer. J. Am. Chem. Soc. 139, 4302-4305 (2017).

13. Dou, C.; Han, L.; Zhao, S.; Zhang, H.; Wang, Y. Multi-Stimuli-Responsive Fluorescence Switching of a Donor-Acceptor $\pi$-Conjugated Compound. J. Phys. Chem. Lett. 2, 666-670 (2011).

14. Yuan, W. Z. et al. Synergy Between Twisted Conformation and Effective Intermolecular Interactions: Strategy for Efficient Mechanochromic Luminogens With High Contrast. Adv. Mater. 25, 2837-2843 (2013).

15. Sagara,Y.; Mutai, T.; Yoshikawa, I.; Araki, K. Material Design for Piezochromic Luminescence: HydrogenBond-Directed Assemblies of a Pyrene Derivative. J. Am. Chem. Soc. 129, 1520-1521 (2007).

16. Davis, D. A. et al. Force-induced Activation of Covalent Bonds in Mechanoresponsive Polymeric Materials. Nature 459, 68-72 (2009).

17. Wang, K. et al. Organic polymorphs: one-compound-based crystals with molecular-conformation- and packing-dependent luminescent properties. Adv. Mater. 26, 6168-6173 (2014).

18. Yoon; S.-J. et al. Multistimuli Two-Color Luminescence Switching via Different Slip-Stacking of Highly Fluorescent Molecular Sheets. J. Am. Chem. Soc. 132, 13675-13683 (2010).

19. Ma, Z.; Teng, Mi.; Wang, Z.; Yang, S.; Jia, X. Mechanically Induced Multicolor Switching Based on a Single Organic Molecule. Angew. Chem. Int. Ed. 52, 12268-12272 (2013).

20. Chung, J. W. et al. Shear- And UV-induced Fluorescence Switching in Stilbenic Pi-Dimer Crystals Powered by Reversible [2 + 2] Cycloaddition. J. Am. Chem. Soc. 131, 8163-8172 (2009).

21. Davis, D. A. et al. Force-induced Activation of Covalent Bonds in Mechanoresponsive Polymeric Materials. Nature 459, 68-72 (2009).

22. Ma, Z. et al. Pressure induced the largest emission wavelength change in a single crystal. Dyes Pigments 162, 136-144 (2019).

23. Wang, Y. et al. Dynamic Behavior of Molecular Switches in Crystal under Pressure and Its Reflection on Tactile Sensing. J. Am. Chem. Soc. 137, 931-939 (2015).

24. Nagura, K. et al. Distinct Responses to Mechanical Grinding and Hydrostatic Pressure in Luminescent Chromism of Tetrathiazolylthiophene. J. Am. Chem. Soc. 135, 10322-10325 (2013).

25. Yuan, H.; Wang, K.; Yang, K. Liu, B. Zou, B. Luminescence Properties of Compressed Tetraphenylethene: The Role of Intermolecular Interactions. J. Phys. Chem. Lett. 5, 2968-2973 (2014).

26. Zhang, S. et al. Rehybridization of Nitrogen Atom Induced Photoluminescence Enhancement under Pressure Stimulation. Adv. Funct. Mater. 27, 1602276 (2014).

27. Gu, Y. et al. Pressure-Induced Emission Enhancement of Carbazole: The Restriction of Intramolecular Vibration. J. Phys. Chem. Lett. 8, 4191-4196 (2017).

28. Gu, Y. et al. Pressure-Induced Emission Enhancement and Multicolor Emission for 1,2,3,4-Tetraphenyl-1,3cyclopentadiene: Controlled Structure Evolution. J. Phys. Chem. Lett. 10, 5557-5562 (2019).

29. Wang, Z. et al. A Novel Mechanochromic and Photochromic Polymer Film: When Rhodamine Joins Polyurethane. Adv. Mater. 27, 6469-6474 (2015).

30. Liu, H. et al. Pressure-Induced Blue-Shifted and Enhanced Emission: A Cooperative Effect Between 
Aggregation-Induced Emission and Energy-Transfer Suppression. J. Am. Chem. Soc. 142, 1153-1158 (2020).

31. Ito, H. et al. Mechanical Stimulation and Solid Seeding Trigger Single-Crystal-To-Single-Crystal Molecular Domino Transformations. Nat. Commun. 4, 2009 (2013).

32. Zhang, G.; Lu, J.; Sabat, M.; Fraser, C. L. Polymorphism and Reversible Mechanochromic Luminescence for Solid-State Difluoroboron Avobenzone. J. Am. Chem. Soc. 132, 2160-2162 (2010).

33. Luo, X. et al. Reversible Switching of the Emission of Diphenyldibenzofulvenes by Thermal and Mechanical Stimuli. Adv. Mater. 23, 261-3265 (2011).

34. Sagara, Y.; Kato, T. Stimuli-responsive Luminescent Liquid Crystals: Change of Photoluminescent Colors Triggered by a Shear-Induced Phase Transition. Angew. Chem. Int. Ed. 47, 5175-5178 (2008).

35. Zhang, Y. et al. Polymorphic crystals and their luminescence switching of triphenylacrylonitrile derivatives upon solvent vapour, mechanical, and thermal stimuli. J. Mater. Chem. C 3, 3049-3054 (2015).

36. Wang, J. et al. Click Synthesis, Aggregation-Induced Emission, E/Z Isomerization, Self-Organization, and Multiple Chromisms of Pure Stereoisomers of a Tetraphenylethene-Cored Luminogen. J. Am. Chem. Soc. 134, 9956-9966 (2012).

37. Feng, H.-T. et al. Selective Host-Guest Co-crystallization of Pyridine-Functionalized Tetraphenylethylenes with Phthalic Acids and Multicolor Emission of the Co-crystals. Chem. Eur. J. 23, 644-651 (2017).

38. Chen, P.-Z. et al. A Solid-State Fluorescent Material Based on Carbazole-Containing Difluoroboron $\beta$ Diketonate: Multiple Chromisms, the Self-Assembly Behavior, and Optical Waveguides. Adv. Funct. Mater. 27, 1700332 (2017).

39. Jiang, M. et al. Multifunctional AIEgens: Ready Synthesis, Tunable Emission, Mechanochromism, Mitochondrial, and Bacterial Imaging. Adv. Funct. Mater. 28, 1704589 (2018).

40. McMillan, P. F. Chemistry of materials under extreme high pressure-high-temperature conditions. Chem. Commun. 919-923 (2014).

41. Sagara, Y.; Kato, T. Brightly Tricolored Mechanochromic Luminescence from a Single-Luminophore Liquid Crystal: Reversible Writing and Erasing of Images. Angew. Chem. Int. Ed. 50, 9128-9132 (2011).

42. Barnett, J. D.; Block, S.; Piermarini, G. J. An Optical Fluorescence System for Quantitative Pressure Measurement in the Diamond-Anvil Cell. Rev. Sci. Instrum. 44, 1-9 (1973).

43. Jayaraman, A. Diamond anvil cell and high-pressure physical investigations. Rev. Mod. Phys. 55, 65 (1983).

44. Wang, L.; Ye, K.Q.; Zhang, H.-Y.; Organic materials with hydrostatic pressure induced mechanochromic properties. Chin. Chem. Lett. 27, 1367-1375 (2016).

45. Yamaguchi, S.; Yoshikawa, I.; Mutaia, T.; Araki, K. Solid-state luminescence of tetraphenylpyrene derivatives: mechano/vapochromic luminescence of 1,3,6,8-tetra(4'-carboxyphenyl)pyrene. J. Mater. Chem. 22, 20065-20070 (2012).

46. Wang, X. et al. Piezochromic luminescence behaviors of two new benzothiazole-enamido boron difluoride complexes: intra- and inter-molecular effects induced by hydrostatic compression. Chem. Commun. 51, 7497-7500 (2015).

47. Gu, Y. et al. Pressure-Induced Emission Enhancement and Multicolor Emission for 1,2,3,4-Tetraphenyl-1,3cyclopentadiene: Controlled Structure Evolution. J. Phys. Chem. Lett. 10, 5557-5562 (2019).

48. Feng, H.-T.; Lam J. W. Y.; Tang, B. Z. Self-assembly of AIEgens. Coord. Chem. Rev. 406, 213142 (2020).

49. Feng, H.-T. et al. Structure, Assembly, and Function of (Latent)-Chiral AIEgens. ACS Mater. Lett. 1, 192-202 (2019).

50. Wang, J. et al. Ionization and Anion- $\pi+$ Interaction: A New Strategy for Structural Design of AggregationInduced Emission Luminogens, J. Am. Chem. Soc. 139, 16974-16979 (2017).

51. Chen, M. et al. Tetraphenylpyrazine-based AIEgens: Facile Preparation and Tunable Light Emission. Chem. Sci. 6, 1932-1937 (2015).

52. Li, W. et al. Fluorescent aggregation-induced emission (AIE)-based thermosetting electrospun nanofibers: 
fabrication, properties and applications. Mater. Chem. Front. 3, 2491-2498 (2019).

53. Niu, G. et al. Specific Two-Photon Imaging of Live Cellular and Deep-Tissue Lipid Droplets by Lipophilic AIEgens at Ultralow Concentration. Chem. Mater. 30, 4778-4787 (2018).

54. Luo, J. et al. Aggregation-induced Emission of 1-methyl-1,2,3,4,5-pentaphenylsilole. Chem. Commun. 21, 1740-1741 (2001).

55. Mei, J. et al. Aggregation-Induced Emission: Together We Shine, United We Soar! Chem. Rev. 115, 11718-11940 (2015).

56. Feng, H.-T.; Yuan, Y.-X.; Xiong, J.-B.; Zheng, Y.-S.; Tang, B. Z. Macrocycles and Cages Based on Tetraphenylethylene With Aggregation-Induced Emission Effect. Chem. Soc. Rev. 47, 7452-7476 (2018).

57. Yang, Z. et al. Recent advances in mechano-responsive luminescence of tetraphenylethylene derivatives with aggregation-induced emission properties. Mater. Chem. Front. 2, 861-890 (2018).

58. Dong, Y. et al. Piezochromic Luminescence Based on the Molecular Aggregation of 9,10-bis((E)-2-(pyrid-2yl)vinyl)anthracene. Angew. Chem. Int. Ed. 51, 10782-10785 (2012).

59. Wang, C. et al. A stable tetraphenylethene derivative: aggregation-induced emission, different crystalline polymorphs, and totally different mechanoluminescence properties. Mater. Horiz. 3, 220-225 (2016).

\section{Acknowledgements}

This project was financially supported by the National Natural Science Foundation of China $(21805002,21788102$ and 21725304), the Research Grants Council of Hong Kong (16305618, 16305518, and C6009-17G), the Innovation and Technology Commission (ITC-CNERC14SC01), Natural Science Basic Research Plan in Shaanxi Province of China (Program 2018JQ2046) and Youths Talents Support Program of Shaanxi Association for Science and Technology (20190610), Shaanxi Provincial Key Laboratory Project (13JS006).

\section{Author contributions}

B. Z. T. and H.-T. F. conceived and designed the experiments. X. W. and C. Q. conducted the synthesis and PL measurement. H. Z. and J. W. performed calculation. Z. F., K. Z. and B. W. performed hydrostatic pressure experiments. H.T. F. and J. W. Y. L. co-wrote the manuscript. X. W. and C. Q. contributed equally to this work.

\section{Competing interests}

The authors declare no competing financial interests.

\section{Additional information}

Supplementary information is available in the online version of the paper. Reprints and permissions information is available online at www.nature.com/reprints.

Correspondence and requests for materials should be addressed to B. Z. T. and H.-T. F. 